\title{
The Relationship between Achievement Goal Orientation of Chinese University Martial Arts Student Athletes with Self-Management, Performance Strategy and Performance
}

\author{
ChengGen Peng ${ }^{1}$ and YanSen $\mathrm{Li}^{2 *}$ \\ ${ }^{1}$ Department, University, Country, Hunan Agricultural University, College of Sport, \\ Changsha, Post code: 410128, China. \\ ${ }^{2 *}$ Department of Physical Education, Woosuk University, 443 Samnye-ro, \\ Samnye-eup, Wanju-gun, Jeollabuk-do, 55338 Republic of Korea \\ 1379569069@qq.com,2*513088839@qq.com
}

\begin{abstract}
This study investigates the differences in achievement goal orientation, self-management, performance strategy, and performance according to demographic characteristics of Chinese university martial arts student athletes. Second, self-management and performance according to achievement goal orientation of Chinese university martial arts student athlete. The purpose is to find out the relationship between strategy and performance. To achieve the purpose of this study, martial arts student athletes from five universities in Hunan Province, China were selected as a population, and a total of 341 copies were selected as final subjects using a convenience sampling method. The collected data was processed as follows by coding the finally selected questionnaire and using the statistical program SPSS Windows 23.0. First, frequency analysis, exploratory factor analysis, reliability verification (cronbach's $\alpha$ ), correlation analysis, and multiple regression analysis were performed for demographic characteristics. First, as a result of examining the relationship between achievement goal propensity and self-management of Chinese university martial arts student athletes, it was found that interpersonal management, a sub-factor of self-management, has an effect on task orientation and self-discipline, which are sub-factors of achievement goal orientation. However, it was found that mental management, a sub-factor of self-management, had an effect on task orientation, a sub-factor of achievement goal orientation. Second, as a result of examining the relationship between achievement goal orientation and performance strategy of Chinese university martial arts student athletes, self-talk and goal setting, which are subfactors of performance strategy, are sub-factors of achievement goal orientation, and condition, which is a sub-factor of performance strategy. It was found to have an effect on task orientation, a sub-factor of achievement goal orientation. Third, as a result of examining the relationship between achievement goal orientation and performance ability of Chinese university martial arts student athletes, it was found that both task orientation and selforientation, which are the sub-factors of achievement goal orientation, affect performance.
\end{abstract}

Keywords: Chinese university, Martial arts student athlete, Achievement goal orientation, Self-management, Performance strategy, Performance

\section{Introduction}

Article history:

Received (January 16, 2021), Review Result (February 20, 2021), Accepted (April 1, 2021) 
Chinese martial arts refer to a systematic training method for mental and physical training, developed from the traditional combat techniques of pre-modern times. It is also referred to as wushu, wudao, or gedouji in ancient China. Strictly defined, it is a word that has a broader meaning than martial arts, weapons, or martial arts, which means the art of fighting. It is not just the art of fighting, but "art"(藝), that is, artistically sublimated. It means having purpose and significance in ordering. Martial arts imply putting more weight on "do-ri", that is, mental discipline. In modern times, they are practically used in almost the same terms without making a big distinction. However, it is clearly distinguished from simply physical fitness or sports. As an aspect of martial arts, it is the fighting sport that focuses on competing and makes it sport [1]. Although martial arts were not adopted as an Olympic sport, the World Championships were held during the Olympics to fully demonstrate the prospects for the globalization of martial arts. At the same time, it is in the process of complementing each other so that martial arts can successfully enter internationalization by taking a well-developed sporting event as an example and using it in the process of internationalization development of martial arts reasonably [2].

Depending on the individual, personality traits define their abilities and success differently, and have a variety of different achievement goals [3]. The most important of these is to show competence or one's own abilities. This perception of competence is classified into a task and an ego-oriented achievement goal according to individual subjective interpretation and judgment. Individuals with task orientation focus on proficiency and learning about the task and evaluate their ability compared to their previous performance [4]. Individuals with selfdirection evaluate their subjective self ability compared to others [5]. These two goal orientations are individual personality traits and are independent and orthogonal concepts related to competence [6]. Most achievement-goal orientation studies classify individual differences in tasks or goals pursuing performance into task-oriented orientation and selforiented orientation, and examine the relationship between motivation, attitude, attribution, anxiety, confidence, psychological skills variables, and exercise performance. [7]. In addition to the achievement goal theory, self-management and performance strategies are recognized as important factors for understanding optimal exercise performance in Korea as well as abroad, and active research is being conducted.

Self-management is a concept similar to the term self-regulation and is used interchangeably, and is used in various aspects such as physical and mental aspects in personal life in training [8]. This is to say that only if there is a process through self-management, the consequences of actions can be brought closer to success. On the other hand, performance strategy is a concept that considers technical, mental, and strategic aspects [10], and it can be seen that it has a great influence to determine the victory or defeat in exercise performance. There are various factors in the performance strategy, condition control that controls all physical, mental, and environmental factors to the best condition, image and goal setting that sets goals by imagination by drawing a game situation as if performing directly in the head, muscle or nerve center Constriction or excitement of the body, relaxation of self-relaxation when tense, control of negative thoughts and positive thoughts so that they can have a good effect, control emotions, and self-talk factors to tell the athletes words that are helpful when performing [11]. Therefore, in this study, the causal relationship between achievement goal orientation, self-management, and performance strategy, which are factors affecting the performance of Chinese university martial arts student athletes, was investigated. to provide the basic information that can help in a meaningful research.

In this study, the following specific research questions were presented [Figure 1]. 


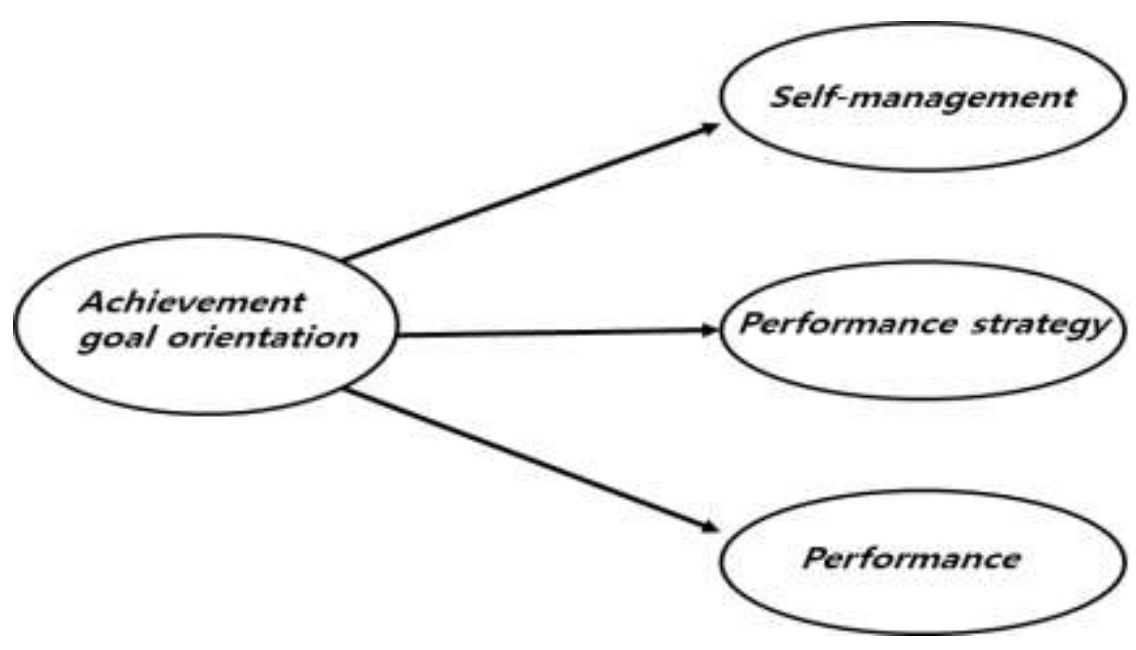

Figure 1. Research model

Research Problem 1. Achievement goal orientation of Chinese university martial arts student athletes will have an effect on self-management.

Research Problem 2. Achievement goal orientation of Chinese university martial arts student athletes will have an effect on performance strategies.

Research Problem 3. Achievement goal orientation of Chinese university martial arts student athletes will have an effect on performance.

\section{Research method}

\subsection{Research subject}

This study is to investigate the relationship between the achievement goal orientation of Chinese university martial arts student athletes' self-management, performance strategy, and performance, in 2020, five universities in Hunan Province, China martial arts student athletes were selected as the population. Samples were extracted using a non-probability sampling method and a convenience sampling method. In order to avoid minimal contact related to COVID-19, the questionnaire was conducted as an Internet survey using an online questionnaire, and the questionnaire was sent by e-mail and mobile phone text messages for about 12 weeks from July to September 2020. Respondents filled in and responded again using the self-administration method. Therefore, a total of 360 respondents who participated in the survey were collected, and a total of 341 copies were selected as final subjects, excluding 19 questionnaires that were judged to be inconsistent or insincere responses.

\subsection{Investigation tool}

In order to measure the relationship between achievement goal orientation and selfmanagement, performance strategy and performance of Chinese college martial arts athletes, achievement goal orientation adopts Duda \& Nicholls (1989) to develop TEOSQ questionnaire, self-management adopts Heo Jung-hoon (2001) to develop ASMQ questionnaire, and performance strategy adopts Seung-mook Oh(2010) Questionnaire used and modified according to the purpose of this research Looking at the specific questionnaire questions, it was composed of a total of 64 questions, including 3 demographic variables, 13 achievement goal orientation, 18 self-management, 24 performance strategies, and 6 competition. 


\subsection{Data processing method}

In this study, factor analysis was conducted to verify the validity of the used items for each variable [11]. To verify the validity of each item, exploratory factor analysis was performed, the suitability of the sample was verified using the Kaiser-Meyer-Olikin index, and Bartlett's unit matrix verification was performed. The factor analysis method used principal component analysis, and the factor rotation method used the Varimax method among the right-angled rotation methods to make the factor classification of variables clearer, and the factors with an eigenvalue of 1 or more and factor loading of .40 or more were included.

For reliability, Chronbach' $\alpha$ was used to confirm the internal consistency between measurement items. In general, if the Chronbach' $\alpha$ coefficient is more than .6 , it can be interpreted that the internal consistency between the measurement items is good. To achieve the purpose of this study, questionnaires that were judged to be insincere and poor reliability were excluded from the analysis, and questionnaires judged to be reliable were coded and analyzed using the statistical program SPSS 23.0 Version.

For demographic characteristics, frequency analysis, exploratory factor analysis to verify validity and reliability, and reliability verification (Cronbach's $\alpha$ ) were conducted. In order to analyze the effect of achievement goal orientation of Chinese university martial arts student athletes on self-management, performance strategy, and performance, independent sample ttest, correlation analysis and multiple regression analysis were conducted to derive the research results.

\subsection{Research limitations}

First, since the subject of this study was limited to martial arts student athletes, the generalized interpretation is somewhat unreasonable due to the limitation of the representativeness of the sample.

Second, caution is required to generalize and apply the results of this study because the sample difference between the groups of the subjects of this study is large.

Third, caution is required because this study did not consider personal physical strength and environmental aspects.

\section{Results}

\subsection{The effect of achievement goal orientation on self-management}

Table 1. Multiple regression analysis of the effect of achievement goal orientation on self-management

\begin{tabular}{|c|c|c|c|c|c|}
\hline \multicolumn{2}{|c|}{ Variable } & B & Std E & $\beta$ & $\mathrm{t}$ \\
\hline \multirow{3}{*}{ Personal management } & (Constant) & 2.043 & .184 & & $8.669 * * *$ \\
\hline & Task orientation & .319 & .093 & .351 & $3.135^{* * *}$ \\
\hline & Magnetism & .238 & .087 & .289 & $2.876^{* *}$ \\
\hline \multicolumn{6}{|c|}{$\mathrm{R}^{2}=.391, \mathrm{~F}=55.991 * * *$} \\
\hline \multirow{3}{*}{ Body care } & (Constant) & 2.166 & .224 & & $9.758 * * *$ \\
\hline & Task orientation & .154 & .098 & .228 & 1.108 \\
\hline & Magnetism & .179 & .099 & .084 & .709 \\
\hline \multicolumn{6}{|c|}{$\mathrm{R}^{2}=.009, \mathrm{~F}=27.135$} \\
\hline
\end{tabular}




\begin{tabular}{|c|c|c|c|c|c|}
\hline \multirow{3}{*}{ Mental management } & (Constant) & 2.054 & .285 & & $7.595 * * *$ \\
\hline & Task orientation & .582 & .121 & .398 & $3.680 * * *$ \\
\hline & Magnetism & .220 & .120 & .217 & 1.955 \\
\hline \multicolumn{6}{|c|}{$\mathrm{R}^{2}=.194, \mathrm{~F}=12.355^{* * *}$} \\
\hline \multirow{3}{*}{ Training management } & (Constant) & 1.874 & .277 & & $6.730 * * *$ \\
\hline & Task orientation & .427 & .138 & .352 & 3.373 \\
\hline & Magnetism & .298 & .126 & .270 & 2.597 \\
\hline \multicolumn{6}{|c|}{$\mathrm{R}^{2}=.065, \mathrm{~F}=2.894$} \\
\hline
\end{tabular}

In order to find out the causal relationship between achievement goal orientation and selfmanagement of Chinese university martial arts student athletes, the results of multiple regression analysis were shown in [Table 1].

First, as a result of analyzing the effect of the sub-factors of achievement goal orientation on interpersonal management, the explanatory power of predicting the relationship between achievement goal orientation and interpersonal management is explained as $39.1 \%\left(\mathrm{R}^{2}=.391\right)$, and the $\mathrm{F}$ value is 55.991. At the level of $\mathrm{p}<.001$, the regression equation was found to be significant, and task orientation $(\mathrm{t}=3.135, \mathrm{p}<.001)$ and self orientation $(\mathrm{t}=2.876, \mathrm{p}<.01)$ were found to be variables that explain interpersonal management.

Second, as a result of analyzing the effect of the sub-factors of achievement goal orientation on mental management, the explanatory power that predicts the relationship between achievement goal orientation and mental management is $19.4 \%\left(\mathrm{R}^{2}=.194\right)$, and the $\mathrm{F}$ value is 12.355. At the level of $p<.001$, the regression equation was found to be significant, and task orientation $(\mathrm{t}=3.680, \mathrm{p}<.001)$ was found to be a variable explaining the interpersonal management.

\subsection{The effect of achievement goal orientation on performance strategy}

Table 2. Multiple regression analysis of the effect of achievement goal orientation on performance strategy

\begin{tabular}{|c|c|c|c|c|c|}
\hline \multicolumn{2}{|c|}{ Variable } & B & Std E & $\beta$ & $\mathrm{t}$ \\
\hline \multirow{3}{*}{ soliloquy } & (Constant) & 1.166 & .631 & & $1.849 * * *$ \\
\hline & Task orientation & .097 & .185 & .072 & .525 \\
\hline & Magnetism & .331 & .153 & .302 & $2.155^{*}$ \\
\hline \multicolumn{6}{|c|}{$\mathrm{R}^{2}=.405, \mathrm{~F}=23.988 * *$} \\
\hline \multirow{3}{*}{ Emotion control } & (Constant) & 1.305 & .693 & & $1.883^{* *}$ \\
\hline & Task orientation & .255 & .204 & .184 & 1.250 \\
\hline & Magnetism & .043 & .169 & .039 & .257 \\
\hline \multicolumn{6}{|c|}{$\mathrm{R}^{2}=.079, \mathrm{~F}=1.330$} \\
\hline \multirow{3}{*}{ Goal setting } & (Constant) & 2.404 & .776 & & $3.096 * * *$ \\
\hline & Task orientation & -.008 & .228 & -.005 & -.034 \\
\hline & Magnetism & .453 & .189 & .359 & $2.397 * *$ \\
\hline \multicolumn{6}{|c|}{$\mathrm{R}^{2}=.393, \mathrm{~F}=21.586^{* *}$} \\
\hline \multirow{2}{*}{ Condition } & (Constant) & 3.199 & .484 & & $6.604 * * *$ \\
\hline & Task orientation & .121 & .142 & .125 & $.852 *$ \\
\hline
\end{tabular}




\begin{tabular}{|c|c|c|c|c|c|}
\hline \multicolumn{7}{|c|}{} & Magnetism & -.004 & .118 & -.005 & -.037 \\
\hline \multirow{3}{*}{ Relax } & $\mathrm{R}^{2}=.374, \mathrm{~F}=31.618^{* *}$ \\
\hline & (Constant) & 3.900 & .610 & & $6.396^{* * *}$ \\
\cline { 2 - 6 } & Task orientation & -.093 & .179 & -.078 & -.517 \\
\cline { 2 - 6 } & Magnetism & .033 & .148 & .034 & .223 \\
\hline \multicolumn{7}{|c|}{$\mathrm{R}^{2}=.041, \mathrm{~F}=.659$} \\
\hline
\end{tabular}

[Table 2] shows the results of multiple regression analysis to find out the causal relationship between achievement goal orientation and performance strategy of Chinese university martial arts student athletes.

First, as a result of analyzing the effect of the sub-factors of achievement goal orientation on self-talk, the explanatory power that predicts the relationship between achievement goal orientation and self-talk is explained as $40.5 \%\left(\mathrm{R}^{2}=.405\right)$, and the $\mathrm{F}$ value is $23.988, \mathrm{p}<<$ At the level of .01, the regression equation was found to be significant, and self-propensity $(\mathrm{t}=2.155, \mathrm{p}<.05)$ was found to be a variable that explains self-talk.

Second, as a result of analyzing the effect of the sub-factors of achievement goal orientation on goal setting, the explanatory power of predicting the relationship between achievement goal orientation and goal setting is $39.3 \%\left(\mathrm{R}^{2}=.393\right)$, and the $\mathrm{F}$ value is 21.586 . At the level of $\mathrm{p}<.01$, the regression equation was found to be significant, and self-propensity $(\mathrm{t}=2.155$, $\mathrm{p}<.01)$ was found to be a variable that explains goal setting.

Third, as a result of analyzing the effect of the sub-factors of achievement goal propensity on condition, the explanatory power of predicting the relationship between achievement goal propensity is $37.4 \%\left(\mathrm{R}^{2}=.374\right)$, and the $\mathrm{F}$ value is $31.618, \mathrm{p}<<$ The regression equation was found to be significant at the .01 level, and self-propensity $(\mathrm{t}=.852, \mathrm{p}<.05)$ was found to be a variable that explains the condition.

\subsection{The effect of achievement goal orientation on performance}

Table 3. Multiple regression analysis of the effect of achievement goal orientation on performance

\begin{tabular}{|c|c|c|c|c|c|}
\hline \multicolumn{2}{|c|}{ Variable } & B & Std E & $\beta$ & $\mathrm{t}$ \\
\hline \multirow{3}{*}{ Performance } & (Constant) & 1.981 & .409 & & $4.839 * * *$ \\
\hline & Task orientation & 174 & .098 & .186 & $1.928 *$ \\
\hline & Magnetism & .315 & .092 & .351 & $3.413 * *$ \\
\hline \multicolumn{6}{|c|}{$\mathrm{R}^{2}=.289, \mathrm{~F}=9.653 * * *$} \\
\hline
\end{tabular}

The results of multiple regression analysis were shown in [Table 3] to find out the causal relationship between the achievement goal orientation and performance of Chinese university martial arts student athletes. As a result of analyzing the effect of the sub-factors of achievement 
goal orientation on performance, the explanatory power that predicts the relationship between achievement goal orientation and performance is $28.9 \%\left(\mathrm{R}^{2}=.289\right)$, and the F value is 9.653, $\mathrm{p}<.001$ At the level, the regression equation was found to be significant, and task orientation $(\mathrm{t}=1.928, \mathrm{p}<.05)$, and self orientation $(\mathrm{t}=3.413, \mathrm{p}<.01)$ were found to be variables that explain the performance.

\section{Conclusion}

The purpose of this study was to investigate the difference in achievement goal orientation, self-management, performance strategy, and performance according to demographic characteristics of Chinese university martial arts student athletes, and the self-management and performance strategy was determined according to the achievement goal orientation of Chinese university martial arts student athletes. The purpose is to derive basic data for efficient human resource management of martial arts, which is one of the types of martial arts, and to create an environment in which athletes can concentrate and to improve performance.

First, as a result of examining the relationship between achievement goal propensity and self-management of Chinese university martial arts student athletes, it was found that interpersonal management, a sub-factor of self-management, has an effect on task orientation and self-discipline, which are sub-factors of achievement goal orientation. However, it was found that mental management, a sub-factor of self-management, had an effect on task orientation, a sub-factor of achievement goal orientation.

Second, as a result of examining the relationship between achievement goal orientation and performance strategy of Chinese university martial arts student athletes, self-talk and goal setting, which are sub-factors of performance strategy, are sub-factors of achievement goal orientation, and condition, which is a sub-factor of performance strategy. It was found to have an effect on task orientation, a sub-factor of achievement goal orientation.

Third, as a result of examining the relationship between achievement goal propensity and performance ability of Chinese university martial arts student athletes, there were no significant differences in all of the sub-factors of achievement goal propensity.

\section{References}

[1] G. Wang and S. Wu, "Chinese martial arts: An idealized martial art," Sports Culture Guide no.20, pp.21-23, Dissertation, Graduate School, Yongin University, (2007)

[2] S. C. Kwon, "The influence of soccer leader's guidance style on achievement goal orientation and exercise commitment," Yongin University Graduate School of Education, (2013)

[3] B. G Jeon and J. C. Hwang, "The influence of junior golf players' achievement goal orientation on sports confidence and exercise commitment," Korean Journal of Sports Science, vol.21, no.6, pp.571-581, (2012)

[4] J. L. Duda, "Goals: A social-cognitive approach to the study of achievement motivation in sport," Handbook of Research on Sport Psychology, pp.421-436, (1993)

[5] J. H. Heo, "Analysis of the relationship between achievement goal orientation and self-management," Physical Science Research, vol.15, no,3, pp.127-135, (2004)

[6] S. Woojin, "The relationship between achievement goal propensity and stress according to perfectionism propensity of college taekwondo athletes," Unpublished master's thesis, Kwandong University Graduate School of Education, (2012)

[7] B. J. Kim, "Measurement of athlete self-management behavior," Physical Science Research, vol.14, no.4, pp.125-140, (2003) 
[8] P. R. Thomas, S. M. Murphy, and L. E. W. Hardy, "Test of performance strategies: Development and preliminary validation of a comprehensive measure of athletes' psychological skills," Journal of Sports Sciences, vol.17, no.9, pp.697-711, (1999)

[9] H. W. Na, "The effect of high school volleyball players' achievement goal orientation on achievement motivation and performance strategy," Unpublished Master's Thesis, Gyeonggi University Graduate School of Education, (2016)

[10] M. R. Kim and D. H. Lee, "The relationship between the achievement goal orientation of boxers and selfmanagement and sports coping strategies," Journal of the Korean Sports Association, vol.48, no.5, pp.139-148, (2009)

[11] B. J. Kim and H. K. Kim, "The effect of achievement goal orientation according to demographic characteristics of boxers on self-management and performance," Journal of the Korean Sports Association, vol.12, no.3, pp.111-124, (2014)

\section{Authors}

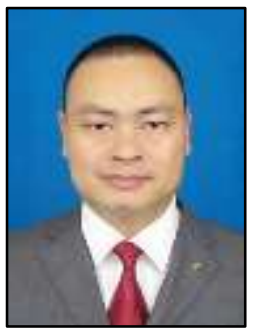

\section{ChengGen Peng}

Chenggen Peng, master's tutor, $\mathrm{PhD}$

Assistant Professor, College of Sport

Research areas: athletic training, traditional sports and sports history

Hunan Agricultural University, Changsha, Hunan, China

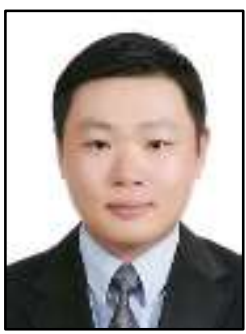

\section{YanSen Li}

Zhengzhou Univ. sports training speciality. B.Ed.

Woosuk Univ. Physical Education Major. M.PE

Woosuk Univ. Physical Education Major. Ph.D. candidate 